\title{
Risk of Dementia in Head Injuries and the Role of Altered Cerebral Blood Flow
}

\author{
Satish Gaddam
}

\begin{abstract}
Traumatic brain injury (TBI) and its consequences on cognitive function has been a long debated topic. In this article, we try to review available literature that tries to explain the consequences of head injury, the change in cerebral flow dynamics and other neurochemical changes that play a role in the development of neurocognitive deficits and other symptoms as a consequence of TBI.
\end{abstract}

Keywords: Dementia; Brain injury; Cognitive deficits; Head Injury; TBI

\section{Cerebral Traumatic Injury (CTI)}

Traumatic brain injury (TBI) is disruption of normal brain function caused by an external mechanical force. Force can be transmitted to the brain from impact to the skull, jaw or body. The constraints of shear force on the brain and blood vessels, which results from acceleration or a sudden and often rotational deceleration of the head, are responsible for most of the damage [1]. TBI can heal completely on its own or produce serious disability or even lead to death. Overall, falls are the main causes of TBI especially in young (under 14 ) and elderly people (65 years old and over). Motor vehicle accidents are the second most common leading causes of TBI and death in young adults. Two-thirds of TBIs occur in men, but lesions of the same severity may develop more unfavorably in women than in men. Participation in sports and being victims of assault are other common causes of head trauma [1].

In the workplace, the causes of TBI are falls, falling of objects, motor vehicle or machine accidents. Certain professions with the most risk are construction, transport, agriculture, forestry, fishing and emergency medical services. Twenty percent of workplace injuries are believed to be attributable to falls on surfaces, uneven or wet or caused by objects out of place, and

Manuscript submitted May 19, 2021, accepted June 8, 2021

Published online June 28, 2021

Baylor Scott and White Centennial, Lone Star Neurology, Frisco, TX, USA. Email: drsatishgaddam@gmail.com

doi: https://doi.org/10.14740/jcs441 they, therefore, appear to be largely preventable. Brain trauma can be either "closed", that is, without lesions of the scalp, or "open", accompanied by lacerations of the scalp. A fractured skull or lacerations of the dura (the outermost and the most resistant of the three membranes separating the skull from the brain) can be associated. There may also be contusions or lacerations of the brain [2]. A closed linear skull fracture is insignificant in itself, except that it is a testament to the intensity of the force exerted on the head. Fractures of closed skullcaps, however, may cause embarrassment (localized depression of the cranial vault) which can damage the brain [2]. Open trauma is a cause of intracranial infections, such as meningitis, subdural empyema and brain abscess. Open trauma can be external or internal. In case of internal open trauma, the skull fracture can affect the paranasal sinuses or mastoid pneumatic cells at the base of the skull and, in combination with damage to the dura mater and arachnoid membrane, serves as bacterial contamination and leads to the aforementioned intracranial infections [3].

\section{What Is Dementia?}

Dementia can be explained as the loss of mental capacity which is considered to be sufficient for undertaking normal routine activities for more than 6 months, not present at the time of birth and not being associated with the alteration and loss of consciousness. Dementia is an umbrella term which covers several types of medical conditions which have common symptoms resulted due to the gradual death of brain cells. Loss within cognitive abilities due to dementia can lead to memory impairment along with drastic changes in behavior [4]. Chronic traumatic encephalopathy (CTE) is referred to be a progressive degenerative disease having a history of receptive brain trauma inclusive of symptomatic concussions and asymptomatic head injuries. Boxers were the first to be the sufferers of this type of dementia. Now CTE is considered to be widespread amongst soccer, football, ice hockey as well as other sports athletes who hold inherent risk of concussive or subconcussive injuries [5]. The word "dementia" is a generic term that groups together more than a hundred diseases that affect brain function. It is mainly the cognitive abilities, such as thinking, memory, orientation in time and space, as well as language, which are affected. As the disease progresses, they are more and more limited in their daily and/ or professional activities and can become dependent on the help of others [6]. 


\section{Dementia or Simple Occasional Forgetfulness!}

We are all subject to occasional forgetfulness, the frequency of which varies according to each one. There is no reason to worry if it happens from time to time that you no longer know whether you have locked the door or lost your glasses. But if you notice that your memory problems are more common and affect more than one area of your cognition, you may want to get it evaluated. That is to say, memory loss is just one symptom of dementia. Depending on its form, dementia can manifest in other ways, such as changes in social behavior [7].

\section{Is There a Cure for Dementia?}

Dementia can have many causes. Some forms of dementia are reversible and can therefore be partially or even completely cured with appropriate treatment. This is the case, for example, with dementia due to a metabolic disease such as hypothyroidism or disorders linked to vitamin B12 deficiency.

Most of the time, however, the brain is directly affected in causes of irreversible dementia. The best known forms of these degenerative brain diseases are Alzheimer's disease (AD) and vascular dementia, Lewy body dementia, frontotemporal dementia and Parkinson's disease dementia. These degenerative forms of dementia cannot be cured or slowed down, with any known therapies. But with the right treatment, those affected can maintain their independence for longer and see their symptoms improve. Provided, however, that the diagnosis has been made as soon as possible by the specialists [8].

\section{Relationship Between Dementia and Head Inju- ries}

Head injuries, depending on their severity and the area of the brain that is affected, can be the cause of cognitive impairment and memory problems similar to those that occur in cases of dementia.

The sequel of injuries of this type can be, among others: vertigo, headache, mood alteration, apathy, depressive or anxiety states, sleep disturbance, and memory problems.

There can be a significant loss of mental abilities in head injury patients (concentration, organizational skills, reasoning skills, decision-making for problem solving, etc.) of paying attention, and of doing things called "executive functions" (planning, organizing, using abstract reasoning, problem solving, and judgment).

In relation to the loss of memory of past and recent events and the acquisition of new memories, amnesia can be reached. Severe head injuries are becoming more common in developed countries. Traffic accidents, the practice of risky sports or work accidents are some of the most frequent causes. However, it is also common for this type of trauma to occur in habitual users of alcohol and other addictive substances [9].

Likewise, it is important to note that some patients with previous head injuries, whether multiple or isolated, develop demen- tia over time, characterized by the existence of problems when expressing themselves and understanding what is said to them (aphasia), the inability to make movements that you are asked to perform (apraxia) or the inability to describe an object (agnosia).

In fact, in studies carried out after the autopsy on people, and even in children, who have suffered serious accidents of this type, it has been found that in $30 \%$ of cases there are amyloid plaques that form shortly after the trauma and that are characteristic of some dementias.

One of the best known types of dementia due to head trauma is the so-called boxer dementia, which is the result of repeated trauma in the exercise of this sport and whose symptoms progress over time. When dementia is the consequence of a single brain injury, it usually does not progress and if it does, one must consider the existence of an additional pathology [10].

More and more data point to the relationship between head injuries and senile dementia. The term "senile dementia" is too broad and vague a concept, which is under review. This contains a significant number of symptoms, with varied origins. Likewise, the word senile, etymologically speaking, refers to old age, or to this period in people's lives. This is related to progressive degeneration and the loss of physical, motor and mental capacities due to advanced age.

Likewise, it applies to degenerative damage produced in the brain, but which can occur before reaching the "third age". In any case, what the specialists are looking for is to give a name and surname to the different symptoms related to dementia, according to their causes and other specificities [11].

\section{Head Injuries: Common Suspects in Senile Dementia}

Any type of head injury can have serious consequences for the person involved, as insignificant as it may seem. The episodes of brain trauma are responsible for a significant number of cases that lead to pathologies. Among these we find AD and Parkinson's disease are more significant.

The problem is that these head injuries seriously affect the lymphatic system, which is responsible for removing toxins from the brain. This exclusive brain cleansing program works during sleep hours. With it, all mental operations are left in optimal conditions to face a new day. A slight impact within this complex network of pipes creates difficulties in the expulsion of waste [12].

The most delicate part of this negative progression is that, unlike most cells in the human body, brain cells do not have regenerative capacities. For this reason, when dying there is no possibility of recovery or replacement. In the absence of adequate cleaning, toxic proteins can accumulate setting the stage for the onset of neurodegeneration.

\section{Role of Inflammation and Thrombosis in TBI}

There is growing evidence between disruption of blood brain barrier and systemic inflammation, altered immune response and increased thrombosis risk in the pathogenesis of TBI. Whether 
these changes persist into the chronic phase of TBI is unknown.

\section{Head Trauma: the Beginning of a Degenerative Process That Takes Time}

Experts assure that fatal consequences on brain cells do not usually occur simultaneously with traumatic episodes. It is a process that can take more time, depending on the severity of the injury itself. However, brain contusions cause immediate damage. They are the beginning of what has been called a "biochemical cascade". This refers to a path that can eventually lead to irreversible states of mental decline [13].

\section{TBI and Development of Dementia and the Role of Changes in Cerebral Flow Dynamics}

TBI has emerged as a very common clinical issue which is also a major source of mortality and morbidity in the current times. Common reasons associated with head injury include falls or accidents. TBIs can be classified as severe, moderate or mild depending upon whether the brain injury has resulted in unconsciousness and its duration as well as severity levels of symptoms [14]. Though, TBIs are usually classified to be mild since they do not pose a life-threatening situation, even this type of TBI can have long-lasting and severe effects. A much feared consequence related to TBI is dementia and several studies indicate that experiencing TBI in early or in mid-life can lead towards the risk of developing dementia [14]. Some common areas related to cognitive impairment that are a result of TBI involve executive and attention functioning, memory information processing speed and others amongst those experiencing that of mild brain injury. Additionally, another observation indicates that repetitive mild brain traumas that are most of the times experienced by professional sportsperson like footballers, hockey players and boxers can be associated with a high degree of CTE. Basically, CTE is a form of dementia having distinct pathologic and clinical features.

Cognitive deficiency in the longer run might incur despite of having a normal looking brain within traditional neuroimaging studies.

The risk of dementia increases with the number and severity of head injuries, according to a large prospective study conducted on a Danish cohort, the results of which are published in the "Lancet Psychiatry". This study was indeed conducted on nearly 2.8 million people, for a total of $27,632,020$ personyears. It shows that people with repeated head trauma have an increase in their risk of dementia by $24 \%$, and the specific risk of $\mathrm{AD}$ increases by $16 \%$. The risk is also increased even with a head injury of moderate severity (concussion), with an increase of $17 \%$ [15].

\section{A Danish Study}

The link between TBI and dementia had already been suggested, mainly for veterans of the wars in Iraq and Afghanistan, and for certain sportsmen (football, American football, hockey, and boxing). But the studies on the subject lacked robustness (too small samples and duration of follow-up, lack of information on the number, severity and date of head injuries, lack of a comparative control population) and the results were contradictory.

This study is the first to follow a sample of sufficient size for a long time. It thus considered in the Danish registers the cases of head trauma that had led to hospitalization or an emergency room visit between 1977 and 2013. The association of the severity, number and date of head injury with the onset of dementia was adjusted for other risk factors such as diabetes, heart disease, depression and drug use [16].

However, other risk factors such as level of education were not taken into account. "It's important to understand how robust this study is" insists a commentary in the same issue of the "Lancet Psychiatry", Carol Brayne of Cambridge University School of Medicine.

\section{A Risk That Increases With the Number of Head Injuries}

Between 1977 and 2013, 4.7\% of the 2.8 million people considered underwent at least one head trauma. And between 1999 and $2013,4.5 \%$ of people over 50 were diagnosed with dementia (with an average age at diagnosis of 80.7 years). The risk of dementia increases with the number of traumatic injuries, from $22 \%$ with a single head trauma to $183 \%$ (almost three times as much) with five or more injuries. The study also found that a single severe head injury increased the risk of dementia by $35 \%$ while a moderate head trauma increased it by $17 \%$.

In addition, the earlier an individual suffers a head injury, the greater the risk of dementia. Gender also has an influence since men are slightly more at risk of developing dementia than women in the same situation. Finally, the study shows that people who have had traumatic fractures outside the sites of the head and neck are not at increased risk of dementia.

Every year, 50 million people around the world experience head trauma, and the main causes are falls, car accidents and assaults.

\section{Classification of TBI and Dementia}

A TBI incurs when an outside force hits hard over the head which is strong enough to cause human brain to violently move within the skull hence disrupting normal functioning of the brain. Depending upon whether the injury resulted in unconsciousness and how long it was, brain injury can be classified into mild injury (mTBI) when there is no loss of consciousness or in case of unconsciousness, it does not last for more than 30 $\mathrm{min}$. Moderate TBI is when the unconsciousness lasts for 30 min where severe brain injury means where unconsciousness exceeds $24 \mathrm{~h}$ [17-19].

\section{Epidemiology}

According to Hyder et al, 2007 [19], almost 10 million people 
across the globe are being affected by TBI on an annual basis. As a matter of fact, this figure is also underestimated as it excludes patients suffering from mTBI who tend to frequently dismiss their symptoms of TBI and fail to seek any type of medical treatment (almost $25 \%$ ) or those who visit private clinics or treat themselves through primary care providers (almost 14\%) and does not report their clinical findings [20, 21]. In an estimation carried out by the Centers for Disease Control and Prevention (CDC), almost 1.7 million Americans tend to suffer from severe TBI which can result in hospitalization, treating in emergency care units or even in deaths on an annual basis [22]. World Health Organization (2015) states that TBI has the potential to surpass various diseases and can become one of the leading causes of disability or death by the year 2020. In a large number of unreported as well as reported cases, TBIs are rendered to be a "silent epidemic" of the world.

In addition to this, Holm et al, 2005 [23], estimated that almost $85-90 \%$ of all treated brain injuries can be categorized as mild which corresponds to an expected incidence of 100 - 300/100,000 cases as per the task force of WHO [23]. The same study proposed that one from every three mTBI victims suffers from long-term cognitive deficiencies [24], which can incur irrespective to normal results obtained from conventional neuroimaging studies. Based upon the data available, a calculation related to the percentage of people having this disease attributed to TBI is between $10 \%$ and $15 \%$ [22].

\section{Etiology of TBI and Dementia}

The most leading causes of TBI amongst the population include motor vehicle accidents, falls, and sports-related injuries as well as assaults [25]. From amongst these falls, are rendered to be the leading cause TBI in every age. However, elderly persons aged 75 years and above reported highest rate of hospitalization due to TBI and hence long-term cognitive impairments or death because of fall. Moreover, other causes identified for TBI include repetitive head trauma from contact sports as well as combat-related injuries in the head amongst soldiers that return from wars [26]. Additionally, brain injuries can also be the result of bullet wounds or any other injury which tends to penetrate within the brain or the skull [26].

Basically, there are two assumptions related to the etiology of TBI where the first is the anterior and frontal cortices susceptible to neural contusion whereas, the second is the rotational and linear forces acting over axon bundles resulting in axonal injury [24, 27].

TBI is rendered to be the most established environmental risk for developing dementia. The recently available data over dementia indicate that: 1) adequate amount of data for the connection between moderate to several TBI and dementia having a higher risk level of developing dementia between two- and fourfold for such patients; 2) scarce evidence is available for the connection between mild TBI associated with the loss of consciousness and developing of dementia; and 3) insufficient or inadequate evidence is available for understanding whether there exists an association between mild level of TBI without losing consciousness and development of dementia [22]. However, no evidence can be traced which indicates that a single episode of mild TBI can increase the risk of dementia. However, in boxers, the risk of developing dementia is deeply associated with the rounds boxed instead of the number of times the boxer was knocked off. This eventually suggests that repetitive mild TBIs which do not result in losing conscious might increase this risk level [28].

\section{Pathophysiology}

Head injury tends to initiate an inflammatory force which releases amino acids like aspartate and glutamate along with free radicals which results in damaging of the tissues [29]. Other potential elements include endogenous opioid peptides like catecholamines, naloxone, thyrotropin-releasing hormone (TRH), acetylcholine, adenosine and lactate along with nitrous oxide [30].

Diffuse axonal injury (DAI) usually incurs after moderate to severe TBI and is an important neuropathological consequence of brain traumas. This aspect involves various abnormalities including direct damaging of the axonal cytoskeleton to that of incurring damage to the disruption of axoplasmic membrane transport, proteolysis, and swelling [31].

Many studies have been undertaken to determine the connection between inheritance of the apolipoprotein $\varepsilon 4$ (APOE $\varepsilon 4$ ) allele and the risk of developing dementia (particularly AD post a head injury). A study carried out in Manhattan [18] people having a history of TBI and the development of APOE $\varepsilon 4$ allele was associated with a 10 -fold increased risk of dementia, whereas APOE $\varepsilon 4$ in the absence of TBI resulted in only a twofold increased risk of dementia. The same study has found no evidence related to developing dementia because of TBI when APOE $\varepsilon 4$ is not present [18].

\section{Pathology}

Within rodent models, brain injury has resulted in neurodegenration as well as progressive brain atrophy which can carry on for at least a year after the occurrence of the [18, 32]. The various types of proteins that are associated with neurodegenrative diseases within humans have also been demonstrated for the accumulation of experimental brain injury in rodents. Amyloid precursor protein is up regulated on an immediate basis after the brain injury and $\beta$-amyloid peptide which accumulates over weeks as well as over months after a trauma [33]. This developed hypothesis holds therapeutic implications in the recent developments within dementia and related diseases and might have a very important role in the management of TBIs [34].

More evidences which indicate that neurodegenration after a head injury may share some similar features with AD in imaging studies. Cerebral atrophy after an injury is not diffused, instead it is regionally selective and those regions where this atrophy is the most prominent after an injury like amygdala, hippocampus, parietal and frontal cortices and precuneus, tend 
to overlap with the regions of predominant $\beta$-amyloid deposition, reduced uptake of glucose as well as progressive atrophy in AD [35]. The findings of these studies can be associated with common molecular mechanisms that are shared between TBI-related neurodegeneration and AD [22]. Though CTE and AD are the distinct elements. Several studies that were made over retired professional athletes who have suffered multiple concussions and have also developed dementia have reported very prominent tau-immunoreactive neurofibrillary tangles (NFTs) and astrocytic tangles; however, $\beta$-amyloid pathology was found in less in than half of the cases $[18,22]$.

\section{Medical Treatment for Dementia in Case of a Brain Injury}

A brain injury which resulted in dementia can benefit from education and emotional support. This can include the following: a detailed discussion of all the different treatment modalities is beyond the scope of this topic: cognitive rehabilitation, behavior modification, family intervention, social services; medications including dopaminergic agents, antidepressants, antipsychotic agents; mood stabilizers, antiepileptic drugs. Other therapeutic strategies for dementia in head injury patients include activity changes, diet, etc.

\section{Conclusion}

The risk of dementia resulted because a brain injury is difficult to predict in certainty. It depends upon multiple factors including the seriousness of the injury. Some may completely recover from severe injuries whereas others might suffer from disabilities for a long time even after milder injuries. Additionally, dementia resulting from a brain injury is different from other forms of dementia. Dementia resulting from a brain injury usually does not worsen with time. Multiple factors including changes in cerebrospinal fluid flow dynamics, alterations in neuro chemicals, sex, age and other comorbidities all play a role in development and recovery of dementia in head trauma patients. Long-term population-based studies are required to further the knowledge base in this specific set of patients.

\section{Acknowledgments}

None to declare.

\section{Financial Disclosure}

None to declare.

\section{Conflict of Interest}

None to declare.

\section{Data Availability}

The author declares that data supporting the findings of the study are available within the article.

\section{References}

1. Centers for Disease Control and Prevention. Injury prevention \& control: traumatic brain injury \& concussion [Accessed Jan 22, 2016]. http://www.cdc.gov/traumaticbraininjury/data/rates.html.

2. Levin HS, Shum D, Chan RC. Understanding traumatic brain injury: current research and future directions. New York (NY): Oxford University Press; 2014.

3. Smith M. Monitoring intracranial pressure in traumatic brain injury. Anesth Analg. 2008;106(1):240-248.

4. Peterson K, Veazie S, Bourne D, Anderson J. In: Evidence Brief: Traumatic Brain Injury and Dementia. Washington (DC), 2019.

5. Dewan MC, Rattani A, Gupta S, Baticulon RE, Hung YC, Punchak M, Agrawal A, et al. Estimating the global incidence of traumatic brain injury. J Neurosurg. 2019;130(4):1080-1097.

6. Fratiglioni L, Paillard-Borg S, Winblad B. An active and socially integrated lifestyle in late life might protect against dementia. Lancet Neurol. 2004;3(6):343-353.

7. Launer LJ. The epidemiologic study of dementia: a lifelong quest? Neurobiol Aging. 2005;26(3):335-340.

8. American Psychological Association. Guidelines for the evaluation of dementia and age-related cognitive change. Am Psychol. 2012;67(1):1-9.

9. Beason-Held LL, Goh JO, An Y, Kraut MA, O'Brien RJ, Ferrucci L, Resnick SM. Changes in brain function occur years before the onset of cognitive impairment. J Neurosci. 2013;33(46):18008-18014.

10. Tran HT, LaFerla FM, Holtzman DM, Brody DL. Controlled cortical impact traumatic brain injury in 3xTg-AD mice causes acute intra-axonal amyloid-beta accumulation and independently accelerates the development of tau abnormalities. J Neurosci. 2011;31(26):9513-9525.

11. Plassman BL, Havlik RJ, Steffens DC, Helms MJ, Newman TN, Drosdick D, Phillips C, et al. Documented head injury in early adulthood and risk of Alzheimer's disease and other dementias. Neurology. 2000;55(8):1158-1166.

12. Crane PK, Gibbons LE, Dams-O'Connor K, Trittschuh E, Leverenz JB, Keene CD, Sonnen J, et al. Association of traumatic brain injury with late-life neurodegenerative conditions and neuropathologic findings. JAMA Neurol. 2016;73(9):1062-1069.

13. Dams-O'Connor K, Gibbons LE, Bowen JD, McCurry SM, Larson EB, Crane PK. Risk for late-life re-injury, dementia and death among individuals with traumatic brain injury: a population-based study. J Neurol Neurosurg Psychiatry. 2013;84(2):177-182.

14. Faul M, Xu L, Wald MW, Coronado VG. Traumatic brain injury in the United States.

15. Savonenko AV, Melnikova T, Hiatt A, Li T, Worley PF, 
Troncoso JC, Wong PC, et al. Alzheimer's therapeutics: translation of preclinical science to clinical drug development. Neuropsychopharmacology. 2012;37(1):261-277.

16. Caye A, Axelrud LK, Salum GA. Traumatic brain injury and dementia. Lancet Psychiatry. 2018;5(10):782-783.

17. Barnes DE, Byers AL, Gardner RC, Seal KH, Boscardin WJ, Yaffe K. Association of mild traumatic brain injury with and without loss of consciousness with dementia in US military Veterans. JAMA Neurol. 2018;75(9):10551061.

18. McKee AC, Cantu RC, Nowinski CJ, Hedley-Whyte ET, Gavett BE, Budson AE, Santini VE, et al. Chronic traumatic encephalopathy in athletes: progressive tauopathy after repetitive head injury. J Neuropathol Exp Neurol. 2009;68(7):709-735.

19. Hyder AA, Wunderlich CA, Puvanachandra P, Gururaj G, Kobusingye OC. The impact of traumatic brain injuries: a global perspective. NeuroRehabilitation. 2007;22(5):341353.

20. Langlois JA, Rutland-Brown W, Wald MM. The epidemiology and impact of traumatic brain injury: a brief overview. J Head Trauma Rehabil. 2006;21(5):375-378.

21. Papa L, Edwards D, Ramia M. Exploring serum biomarkers for mild traumatic brain injury. In: Kobeissy FH, editor. Brain neurotrauma: molecular, neuropsychological, and rehabilitation aspects. Boca Raton (FL): CRC Press; 2015. Chapter 22.

22. Shively S, Scher AI, Perl DP, Diaz-Arrastia R. Dementia resulting from traumatic brain injury: what is the pathology? Arch Neurol. 2012;69(10):1245-1251.

23. Holm L, Cassidy JD, Carroll LJ, Borg J, Neurotrauma Task Force on Mild Traumatic Brain Injury of the, W. H. O. Collaborating Centre. Summary of the WHO Collaborating Centre for neurotrauma task force on mild traumatic brain injury. J Rehabil Med. 2005;37(3):137-141.

24. Buki A, Povlishock JT. All roads lead to disconnection?-Traumatic axonal injury revisited. Acta Neurochir (Wien). 2006;148(2):181-193; discussion 193-184.

25. Wintermark M, Sanelli PC, Anzai Y, Tsiouris AJ, Whitlow CT, Institute ACRHI, Institute ACRHI. Imaging evi- dence and recommendations for traumatic brain injury: conventional neuroimaging techniques. J Am Coll Radiol. 2015;12(2):e1-14.

26. Okie S. Traumatic brain injury in the war zone. N Engl J Med. 2005;352(20):2043-2047.

27. Meythaler JM, Peduzzi JD, Eleftheriou E, Novack TA. Current concepts: diffuse axonal injury-associated traumatic brain injury. Arch Phys Med Rehabil. 2001; 82(10):1461-1471.

28. Lee B, Newberg A. Neuroimaging in traumatic brain imaging. NeuroRx. 2005;2(2):372-383.

29. Morganti-Kossmann MC, Rancan M, Stahel PF, Kossmann T. Inflammatory response in acute traumatic brain injury: a double-edged sword. Curr Opin Crit Care. 2002;8(2):101-105.

30. Leker RR, Shohami E. Cerebral ischemia and trauma-different etiologies yet similar mechanisms: neuroprotective opportunities. Brain Res Brain Res Rev. 2002;39(1):5573.

31. Johnson VE, Stewart W, Smith DH. Axonal pathology in traumatic brain injury. Exp Neurol. 2013;246:35-43.

32. Bramlett HM, Dietrich WD. Quantitative structural changes in white and gray matter 1 year following traumatic brain injury in rats. Acta Neuropathol. 2002;103(6):607614.

33. Iwata A, Chen XH, McIntosh TK, Browne KD, Smith $\mathrm{DH}$. Long-term accumulation of amyloid-beta in axons following brain trauma without persistent upregulation of amyloid precursor protein genes. J Neuropathol Exp Neurol. 2002;61(12):1056-1068.

34. Uryu K, Laurer H, McIntosh T, Pratico D, Martinez D, Leight S, Lee VM, et al. Repetitive mild brain trauma accelerates Abeta deposition, lipid peroxidation, and cognitive impairment in a transgenic mouse model of Alzheimer amyloidosis. J Neurosci. 2002;22(2):446-454.

35. Buckner RL, Sepulcre J, Talukdar T, Krienen FM, Liu $\mathrm{H}$, Hedden T, Andrews-Hanna JR, et al. Cortical hubs revealed by intrinsic functional connectivity: mapping, assessment of stability, and relation to Alzheimer's disease. J Neurosci. 2009;29(6):1860-1873. 